\title{
Successful management of recurrent Actinomyces esophagobronchial fistula with self-expanding covered esophageal stent
}

\author{
Hyde M. Russell, MD, Alberto L. de Hoyos, MD, and Matthew G. Blum, MD, Chicago, III
}

$\mathrm{F}$ istulas between the esophagus and tracheobronchial tree have been effectively managed with covered stenting of either the esophagus or the tracheobronchial tree. Ideally, stents for benign fistulas would remain in the position in which they were placed and would be easily removable after fistula closure. Unfortunately, in nonstenotic disorders, stent migration has posed a significant problem. ${ }^{1}$ We describe the use of a new covered removable esophageal stent (Figure 1) that is designed not to migrate in the treatment of a benign fistula.

\section{Clinical Summary}

A 59-year-old woman with diabetes and reflux had a productive cough that persisted for several months despite treatment with broad-spectrum antibiotic therapy. Eventually, she noticed that her cough was associated with swallowing. Evaluation with a bronchoscope revealed a bronchoesophageal fistula. Cultures from the fistula biopsy specimens grew Actinomyces. The communication between the bronchus intermedius and the esophagus was confirmed with an esophagoscope and a barium esophagogram. After 2 weeks of treatment with penicillin, she underwent a right thoracotomy with division of the fistula and interposition of an intercostal muscle flap. A postoperative esophagogram revealed no leak or fistula, and the patient was started on a regular diet. Her symptoms of coughing with swallowing recurred 2 weeks later, prompting a subsequent swallow study that confirmed recurrent fistulization (Figure 2, $A$ ). She was referred to our institution for further management, at which time she was unable to drink thick or thin liquids owing to severe coughing after swallowing.

Given her recent operation and the concern for extensive inflammation and adhesions, she was treated with a removable esophageal stent for 6 weeks. A $22 \mathrm{~mm} \times 7 \mathrm{~cm}$ hybrid (polyurethane line nitinol) self-expanding stent (Alveolus, Inc, Charlotte, NC) was inserted under fluoroscopic guidance after localizing the fistula with a bronchoscope and esophagoscope. A postoperative barium swallow study showed that the fistula had

From the Department of Thoracic Surgery, Northwestern Memorial Hospital, Chicago, Ill.

Received for publication April 19, 2007; accepted for publication May 22, 2007.

Address for reprints: Matthew G. Blum, MD, 201 E. Huron, Galter 10-105, Chicago, IL 60611 (E-mail: mblum@nmh.org).

J Thorac Cardiovasc Surg 2007;134:1086-7

$0022-5223 / \$ 32.00$

Copyright $\odot 2007$ by The American Association for Thoracic Surgery doi:10.1016/j.jtcvs.2007.05.055 been successfully excluded. The patient, however, had difficulty swallowing and reported having chest and back pain after the procedure. Two days later the stent was removed without difficulty and replaced with an $18 \mathrm{~mm} \times 10 \mathrm{~cm}$ stent. A percutaneous gastrostomy was placed in the same setting for supplemental enteral feeding access. This final procedure was well tolerated, excluded the fistula by esophagogram (Figure 2 , $B)$, and relieved her symptoms. The patient was discharged home on a regimen of soft foods and tube feedings. She continued penicillin treatment for actinomycosis.

Six weeks after stent placement, the patient was returned to the operating room for stent removal. Endoscopic examination showed the stent in good position, without migration, and the fistula remained excluded. It was removed easily with biopsy forceps through the flexible esophagoscope. There was a small amount of granulation tissue in the esophagus where the distal edge of the stent abutted the mucosa. The remainder of the esophagus was unremarkable, and the point of prior fistualization appeared healed. A swallow study revealed no evidence of bronchoesophageal communication. Ten days after stent removal she reported occasional slight coughing with thin liquids. An esophagogram showed a small recurrent fistula. She was maintained on soft foods and thick liquids. Two weeks later she noted cessation of coughing with liquids, and an esophagogram confirmed fistula closure (Figure 2,C). Her G-tube was removed. She continues to have normal swallowing and no symptoms of fistulization 5 months after stent removal.

\section{Discussion}

The stent used in this case was ideally suited for this particular patient, who required stenting for an intermediate duration (6-8 weeks). Excluding the fistula with an internally lined stent not only allows for a delay of reoperation to a more favorable time distant from the initial operation, but in this case it eliminated a second operation altogether by allowing the fistula to close. The Alveolus self-expanding covered stent is a new addition to the growing set of tools available for endoscopic esophageal interventions. The stent structure is unique in that an outer self-expanding nitinol cage is lined on the inside by polyurethane. The metal tips act like scales that provide superficial fixation and prevent distal migration. A polyester ring suture on

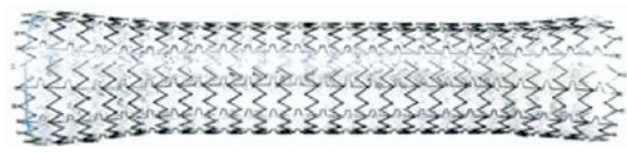

Figure 1. Polyurethane-lined nitinol cage stent. Suture at mouth of left (proximal) end is designed for grasping to facilitate stent removal. 

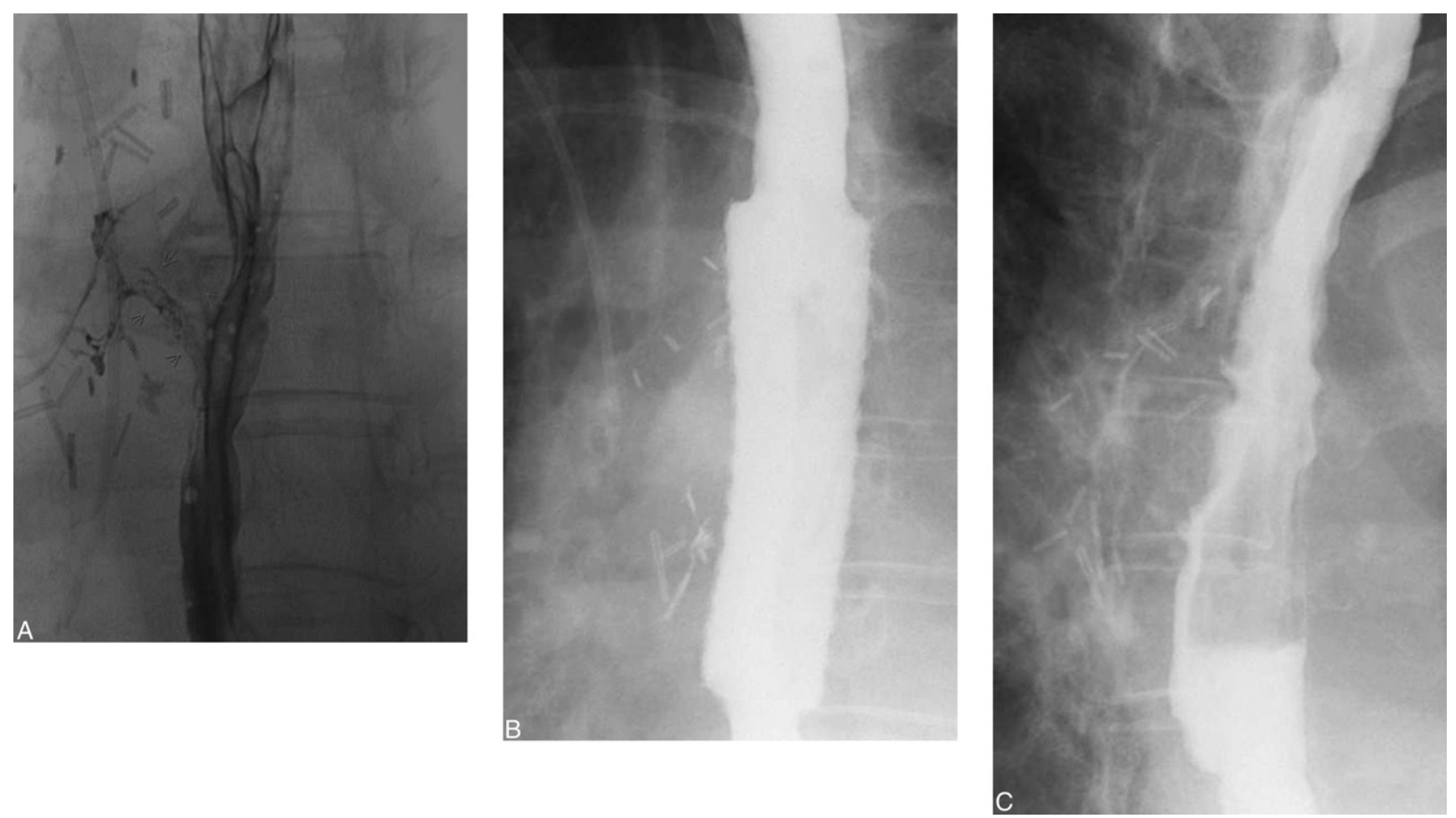

Figure 2. Scan of esophagus showing esophagobronchial fistula (A), stent covering fistula (B), and esophagogram after fistula closure (C).

the proximal end is designed to be grasped by biopsy forceps for easy removal. Granulation tissue formation has been a problem with uncovered stents and the bare metal ends of covered stents. Laboratory studies ${ }^{2}$ and our experience suggest that granulation tissue formation may be less of a problem with this new stent. It is a reasonable alternative to surgical repair in the initial treatment of acquired tracheal or bronchoesophageal fistulas and can serve as a temporizing measure if not a definitive treatment.

\section{References}

1. Pennathur A, Chang AC, McGrath KM, Steiner G, Alvelo-Rivera M, Awais $\mathrm{O}$, et al. Polyflex expandable stents in the treatment of esophageal disease. University of Pittsburgh Medical Center, Pittsburgh (PA); University of Michigan, Ann Arbor (MI). Abstract presented at the 43rd annual Society of Thoracic Surgeons meeting in San Diego, CA, January 30, 2007.

2. Baron TH, Burgart LJ, Pochron NL. An internationally covered (lined) self-expanding metal esophageal stent: tissue response in a porcine model. Gastrointest Endosc. 2006;64,2:263-267. 